\title{
A Multi-Channel Gas Sensor Using Fabry-Perot Interferometer-Based Infrared Spectrometer
}

\author{
Ju Chan Choi1 ${ }^{1}$ June Kyoo Lee'1, and Seong Ho Kong1,2,+
}

\begin{abstract}
We report a Fabry-Perot interferometer (FPI)-based multi-channel micro-spectrometer used for multi-gas measurement in the spectral range of 3-5 $\mu \mathrm{m}$ and its gas sensing performance. The fabricated infrared (IR) spectrometer consists of two parts: an FPI on the top side for selective IR filtering and a $\mathrm{V}_{2} \mathrm{O}_{5}$-based IR detector array on the bottom side for the detection of the filtered IR. Experimental results show that the FPI-based multi-channel gas sensor has reliability and selectivity for simultaneously detecting environmentally harmful gases such as $\mathrm{CH}_{4}, \mathrm{CO}_{2}, \mathrm{~N}_{2} \mathrm{O}$ and $\mathrm{CO}$ in the spectral range of 3-5 $\mu \mathrm{m}$. The fabricated FPI-based multi-channel gas sensor also demonstrated that a reliable and selective detection of gas concentrations ranging from 0 to $500 \mathrm{ppm}$ is feasible. In addition, the electrical characteristics demonstrate a superior response performance in regards to the selectivity in the multi-target gases.
\end{abstract}

Keywords : Fabry-Perot interferometer, Multi-channel infrared gas sensor, MEMS

\section{INTRODUCTION}

Many researchers have striven to develop long lasting stable low cost infrared (IR) gas detectors for toxic and environmental pollutant detection. IR gas detectors offer significantly useful characteristics compared to other sensors, especially in their selective gas absorption and reliable optical response. However, many gaseous species have their absorption spectra in an identical spectral region, which makes it difficult to distinguish individual gas species when simultaneously detecting various target gases. As a result, a multi-gas measurement system is needed to meet the requirements for high absorption resolution, species selectivity, and band sensitivity from the near-infrared (NIR) to the mid-infrared (MIR) ranges. Consequently, many different types of Fabry-Perot interferometer (FPI)-based gas sensors and sensor systems

${ }^{1}$ Graduate School of Electrical Engineering and Computer Science, IT3213A, Kyungpook National University, 1370 Sangyuk-dong, Buk-gu, Daegu 702-701, Korea

${ }^{2}$ School of Electronics Engineering, College of IT Engineering, IT3-213, Kyungpook National University, 1370 Sangyuk-dong, Buk-gu, Daegu 702-701, Korea

${ }^{+}$Corresponding author: shkong@knu.ac.kr

(Received : Jun. 26, 2012, Revised : Sep. 7, 2012, Accepted : Sep. 12, 2012)

This is an Open Access article distributed under the terms of the Creative Commons Attribution Non-Commercial License(http://creativecommons.org/licenses/bync/3.0)which permits unrestricted non-commercial use, distribution, and reproduction in any medium, provided the original work is properly cited. have been developed [1-5]. Makoto et al have reported upon an electro-statically driven tunable FP filter with a wide wavelength range for more precise selective gas sensing [4]. Hui, Carlos, and Vargas et al have presented a fiber FP gas system that measures mixed gas species with a high resolution gas filter utilizing a surface micromachining process [5]. In addition, there are other examples regarding the use of FP filter-based gas sensors and systems [6-9]. In this paper, we present a FPI-based multichannel IR micro-spectrometer used for multi-gas measurement and its gas sensing performance. The FP filter is used as a key component in the sensor reported in this paper and greatly simplifies the construction of the gas sensor. As a result, a simple and highly selective gas sensor is realized in this work.

\section{DESIGN}

\subsection{Structure of the proposed gas sensor}

Fig. 1 presents the structure of the proposed gas sensor. The proposed multi-channel gas sensor functionally consists of a FPI array on top for selective IR filtering and a corresponding $\mathrm{V}_{2} \mathrm{O}_{5}$-based bolometric IR detector array underneath each FPI filter for the sensing of the filtered IR spectrum. Both the FPI and IR detector are prepared on a double-layer membrane $\left(\mathrm{Si}_{3} \mathrm{~N}_{4} / \mathrm{SiO}_{2}\right)$ for minimizing both 
the parasitic thermal loss generated by thermal conduction and the internal stress of the membrane.

The proposed FPI consists of two parallel mirrors, upper silver (Ag) and lower aluminum (Al) layers, and an etalon layer of silicon oxide sandwiched in between the two mirrors. A specific resonance wavelength is selected by different FPI etalon thicknesses.

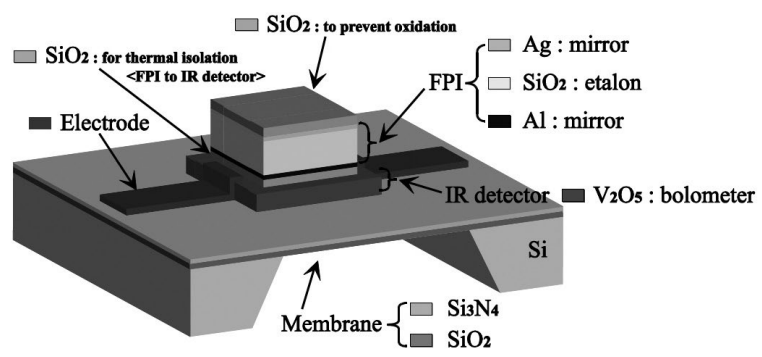

Fig. 1. Schematic view of the FPI-based IR spectrometer.

The bolometric IR detectors are prepared using vanadium oxide, which is one of the uncooled type IR detectors. A bolometer can detect the presence of IR by the increment or decrement in resistance due to the increment of temperature by IR illumination. A fuller description of the operating principles and IR detection mechanism are described in our previously published paper [10].

Fig. 2 shows the schematic view of the multi-channel gas sensor using the FPI-based IR spectrometer. A stacked pair of FPI and IR detectors placed in multi-arrays is a unit component used to detect a single target gas. By preparing the gas sensing unit in the form of an array, it is possible to simultaneously detect the various target gases.

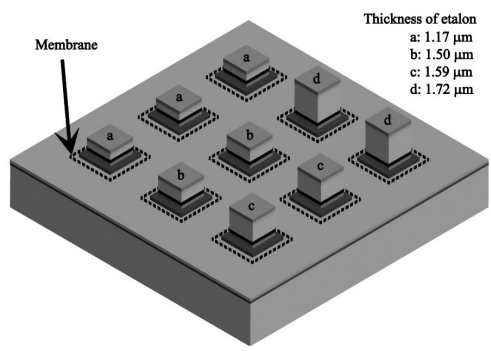

Fig. 2. Schematic view of the multi-channel gas sensor using the FPI-based IR spectrometer.

\subsection{Multi-channel FPI}

Fig.3 illustrates the simulated FP filtering characteristics as a function of the etalon thickness. In the simulation, 30 $\mathrm{nm}$-thick $\mathrm{Ag}$ and $\mathrm{Al}$ are used as the top and bottom mirrors with different $\mathrm{SiO}_{2}$ interlayer thicknesses of 1.17, 1.50, 1.59 and $1.72 \mu \mathrm{m}$, used to filter the wavelength range for the target gases. In this work, the spectral range of 3 to $5 \mu \mathrm{m}$ wavelength was scanned and measured for the detection of specific target gases, $\mathrm{CH}_{4}, \mathrm{CO}_{2}, \mathrm{~N}_{2} \mathrm{O}$ and $\mathrm{CO}$. The simulated FPI result demonstrates good wavelength filtering characteristics as a function of etalon thickness.

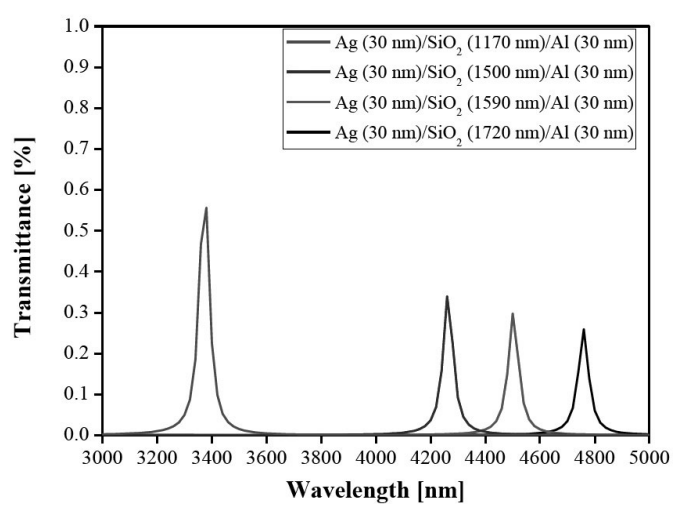

Fig. 3. Simulation results of the FP filter as a function of etalon thickness.

Fig. 4 shows the fabricated FP filter characteristics as a function of the etalon thickness measured in order to verify the simulated results. Each FP filter is made on a bare glass substrate for the optical measurement of the FPI-based filter. The results of the fabricated FPI filter were quite consistent with the simulation results, as can be seen in Fig. 4 The discrepancy between the simulated and measured characteristics of the FP filter is mainly due to the nonuniformity in the IR incident angle and variation in the thickness of the thin film.

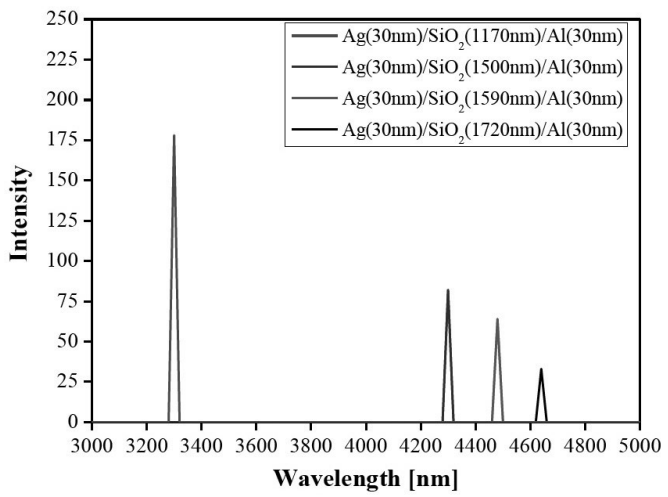

Fig. 4. Measurement results of the FP filter as a function of the $\mathrm{SiO}_{2}$ etalon thickness 


\section{FABRICATION}

Fig. 5 shows the fabrication sequence of the FPI-based IR spectrometer. The $\mathrm{SiO}_{2}$ layer was grown by wet oxidation, and the $\mathrm{Si}_{3} \mathrm{~N}_{4}$ layer was deposited by PECVD. Vanadium oxide is deposited using an RF sputtering system and patterned using a Cr7SK solution. In order to achieve high performance characteristics in an IR detector, it requires a thermo-sensing film with a high TCR value, low resistance, low noise, and a fast response time. We selected the vanadium oxide thin films as the thermal sensing layer in the un-cooled IR detector for their large TCR, superior electrical resistance, and low 1/f noise. In order to reduce the undesirable electrical parameters, an added annealing process was introduced. The measured TCR value of as-deposited vanadium oxide layer without annealing was approximately $-0.9 \% / \mathrm{K}$, which is a rather low value. However, a high TCR value about $-4 \% / \mathrm{K}$ was achieved after performing an annealing process for $2 \mathrm{~min}$ at $500^{\circ} \mathrm{C}$. The details of the annealing process can be found in our previously published paper [10]. The measurement results show a good TCR value as well as appreciable electrical resistances suitable for IR sensor applications.
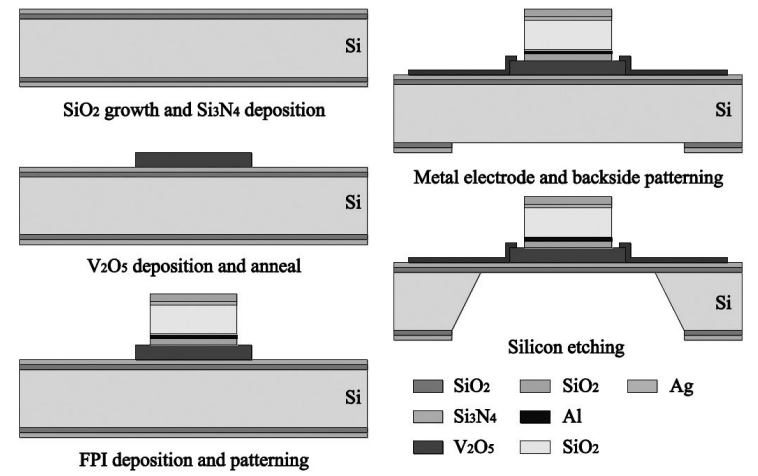

Fig. 5. Fabrication sequence for the FPI-based IR spectrometer.

The FPI filter layers, $\mathrm{Ag} / \mathrm{SiO}_{2} / \mathrm{Al}$, are then deposited on the front side of the composite membrane by e-beam sputtering and patterned by wet etching. A PECVD oxide layer is used as the etalon layer between the two metal mirrors. The thickness of the etalon layer is controlled during the fabrication process. The oxide layer between the FPI and the IR detector is used for thermal isolation between the FPI and IR detector. The top oxide layer on the FPI keeps the Ag layer from oxidation.

The bulk silicon on the backside is selectively etched away using $25 \mathrm{wt} \%$ tetramethyl ammonium hydroxide
(TMAH) solutions in order to minimize the heat loss. The fabricated multi-channel gas sensor using the FPI-based IR spectrometer is shown Fig. 6.

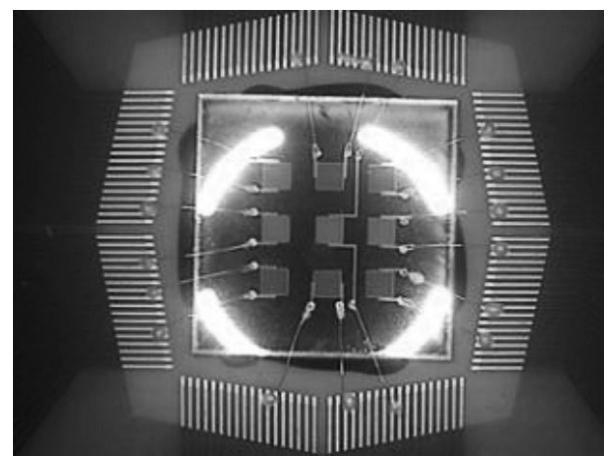

Fig. 6. Fabricated multi-channel gas sensor using the FPI-based IR spectrometer.

\section{MEASUREMENT}

The spectral response of the fabricated FPI-based IR spectrometer was measured with the equipment set-up shown in Fig. 7. Optical systems often require the use of a chopper to modulate the light beam. A mechanical optical chopper (Model SR540) was used for square-wave modulation of the optical signal intensity. The unit is capable of chopping light sources at rates of $4 \mathrm{~Hz}$ to 3.7 $\mathrm{kHz}$. The chopper frequency was set to $800 \mathrm{~Hz}$. The output characteristics of the fabricated device were measured using a lock-in amplifier using the supplied chopper frequency and current signal. A commercialized IR lamp (72-0100-08 IR source BHK (MAX-IR)) was used for the IR source part. Finally, the measured radiation wavelength range at the exit slit of the monochrometer is 1 to $6 \mu \mathrm{m}$ with a $54 \mathrm{~mW} / \mathrm{cm}^{2}$ flux density.

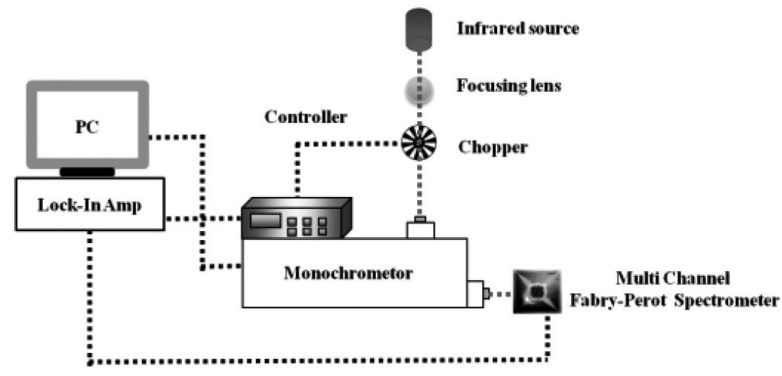

Fig. 7. The measurement set-up used to analyze the FPI-based IR spectrometer. 
Measurement of the proposed multi-channel gas sensor was conducted by a scanning wavelength within 3 to $5 \mu \mathrm{m}$ at a resolution of $20 \mathrm{~nm}$.

Fig. 8 presents the measurement results of the output voltage as a function of the $\mathrm{SiO}_{2}$ etalon thickness on the fabricated FP-based IR spectrometer. The fabricated IR spectrometer shows the proper output voltage at 3.3, 4.32, 4.5 and $4.62 \mu \mathrm{m}$ peaks that correspond to the wavelengths where $\mathrm{CH}_{4}, \mathrm{CO}_{2}, \mathrm{~N}_{2} \mathrm{O}$ and $\mathrm{CO}$ gases have their maximum absorption, respectively.

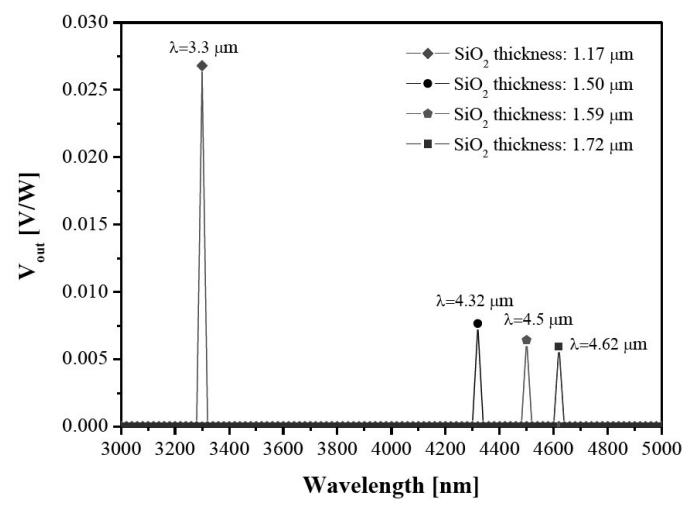

Fig. 8. The output voltage characteristic of the FPI-based IR spectrometer as a function of the $\mathrm{SiO}_{2}$ etalon thickness.

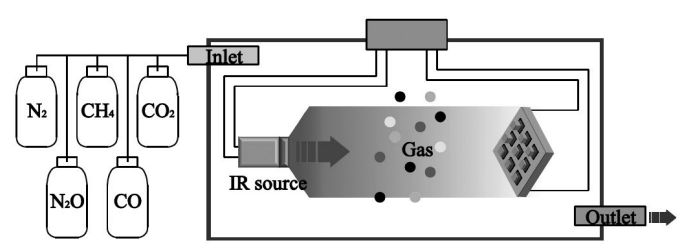

Fig. 9. The gas measurement set-up used to analyze the FPI-based IR spectrometer.

Fig. 9 and 10 show the gas measurement system and resistance changes of the fabricated IR spectrometer-based gas sensor for $\mathrm{CO}, \mathrm{CO}_{2}, \mathrm{~N}_{2} \mathrm{O}$ and $\mathrm{CH}_{4}$ gases depending on their concentrations. The FPI-based IR filter selects a specific wavelength that corresponds to a single target gas and the filtered wavelength spectrum arrives at the IR detector. The measurement results revealed that the fabricated bolometric IR detector shows a different output resistance depending on the concentration of the gas concentration.

Fig. 11 displays the gas absorption characteristics of the fabricated FPI-based multi-gas sensor as a function of the target gas concentrations. The measurement results show superior response characteristics for $\mathrm{CH}_{4}, \mathrm{CO}_{2}, \mathrm{~N}_{2} \mathrm{O}, \mathrm{CO}$ gases at concentrations ranging from 0 to $500 \mathrm{ppm}$. The proposed IR spectrometer-based multi-gas sensor is able to selectively detect different gases simultaneously on a single chip.

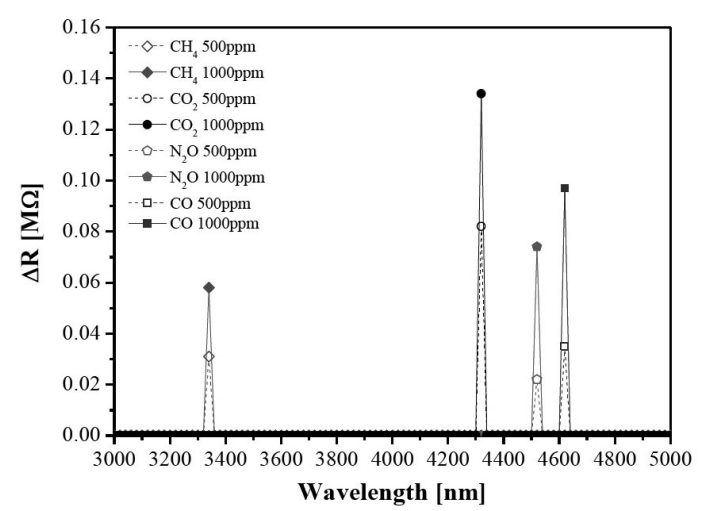

Fig. 10. The resistance changes of the FPI-based IR spectrometer for $\mathrm{CH}_{4}, \mathrm{CO}_{2}, \mathrm{~N}_{2} \mathrm{O}$, and $\mathrm{CO}$ gases at 500 and $1000 \mathrm{ppm}$ concentrations.

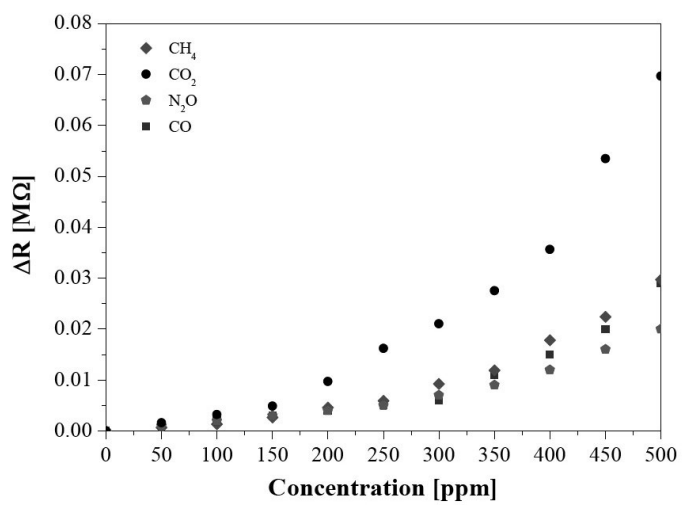

Fig. 11. The resistance change of the FPI-based IR spectrometer as a function of multi-gas concentrations.

\section{CONCLUSIONS}

An FPI-based multi-channel IR gas sensor has been designed and fabricated using micro electro mechanical systems (MEMS) technology and its gas sensing performance with $\mathrm{CH}_{4}, \mathrm{CO}, \mathrm{N}_{2} \mathrm{O}$ and $\mathrm{CO}_{2}$ gases have been characterized. The proposed sensor could simultaneously detect the various target gases, $\mathrm{CH}_{4}, \mathrm{CO}, \mathrm{N}_{2} \mathrm{O}$ and $\mathrm{CO}_{2}$ on a single sensor chip. Furthermore, more kinds of gas detection can be realized by the proposed method as etalon thickness engineering on the proposed multi-channel IR gas sensor could control the filtering performance of the target gases. 


\section{ACKNOWLEDGEMENTS}

This research was supported by Kyungpook National University Research Fund, 2012.

\section{REFERENCES}

[1] D. Cristea, M. Kusko, R. Muller, E. Manea, and D. Syvridis, "Design and experiments for tunable optical sensor fabrication using (111)-oriented silicon micromachining", Sens. Actuators A, Vol. 113, pp. 312318, 2004.

[2] A. T. T. D. Tran, Y. H. Lo, Z. H. Zhu, D. Haronian, and E. Mozdy, "Surface micromachined Fabry-Perot tunable filter", IEEE Photonic Tech Letters, Vol. 8, pp. 393-395, 1996.

[3] L. y. Lin, J. L. Shen, M. C. Uw, and A. M. Sergent, "Tunable three-dimensional solid Fabry-Perot etalons fabricated by surface-micromachining", IEEE Photonic Tech Letters, Vol. 8, pp. 101-103, 1996.

[4] M. Noro, K. Suzuki, N. Kishi, H. Hara, T. Watanabe, and $\mathrm{H}$. Iwaoka, " $\mathrm{CO}_{2} / \mathrm{H}_{2} \mathrm{O}$ gas sensor using a tunable Fabry-Perot filter with wide wavelength range", Proc. of IEEE conf. on Micro Electro Mechanical Systems, pp. 319-322, Kyoto, Japan, 2003.
[5] C. Calaza, L. Fonseca, M. Moreno, S. Marco, C. Cane, and I. Gracia, "A surface micromachining process for the development of a medium-infrared tunable FabryPerot interferometer", Sens. Actuators A, Vol. 113, pp. 39-47, 2004.

[6] J. P. Dakin, H. O. Edwards, and B. H. Weigl, "Progress with optical gas sensors using correlation spectroscopy", Sens. Actuators B, Vol. 29, pp. 87-93, 1995.

[7] M. blomberg, A. Torkkeli, A. Lehto, C. Helenelund, and M. Viitasalo, "Electrically tunable micromachined fabry-perot interferometer in gas analysis", Physica Scripta, Vol. 69, pp. 119-121, 1997.

[8] E. Vargas and H. N. Rutt, "Design of $\mathrm{CO}, \mathrm{CO}_{2}$ and $\mathrm{CH}_{4}$ gas sensors based on correlation spectroscopy using a Fabry-Perot interferometer", Sens. Actuators B, Vol. 137, pp. 410-419, 2009.

[9] H. Ding, j. Liang, J. Cui, and X. Wu, "A novel fiber Fabry-Perot filter based mixed-gas sensing system", Sens. Actuators B, Vol. 138, pp. 154-159, 2009.

[10] J. C. Yang, H. Jung, G. J. Lee, J. Y. Kang, J. G. Koo, J. M. Park, K. S. Park, and S. H. Kong, "Microelectro-mechanical-systems-based infrared spectrometer composed of multi-slit grating and bolometer array", Jpn. J. Appl. Phys., Vol. 47, pp. 6943-6948, 2008.

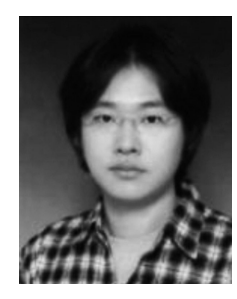

Ju Chan Choi received his B.S. and M. Sc. degrees in electrical engineering and computer science from Kyungpook National University, Korea, in 2007 and 2009, respectively. In 2009, he joined the Micro/Nano System Technology Laboratory in the School of Electrical Engineering and Computer Science, Kyungpook National University, where he is currently working as a $\mathrm{Ph}$. D candidate. His research interests include MEMS-based sensors and power MEMS fields.

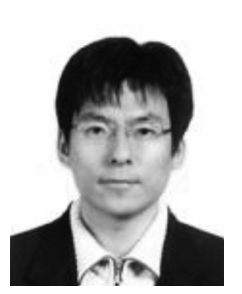

June Kyoo Lee received the B.S. and M.S. degrees in Metallurgical Engineering and Sensor Engineering from Kyungpook National University, Korea, in 2001 and 2003, respectively. In 2008, he joined the Micro/Nano System Technology Laboratory in the School of Electrical Engineering and Computer Science, Kyungpook National University, where he is currently working as a $\mathrm{Ph}$. D candidate. His research interests include electrowetting physics, MEMS-based sensors and actuators, and MEMS-based energy harvesting devices. 
Seong Ho Kong received his B.S. degree in electronic engineering from Kyungpook National University, Korea, in 1993 and his M.Sc. degree in mechatronics and precision engineering from Tohoku University, Japan, in 1996. In 2002, he received his Ph.D. degree in electrical engineering from Delft University of Technology, the Netherlands, with his thesis work concerning infrared micro-spectrometers, and joined ASM Europe as a senior process engineer. In 2004, he joined the School of Electronics Engineering, Kyungpook National University, Korea. His main research activities currently involve the design and fabrication of sensors/actuators-based on MEMS technology. 